\title{
OPEN Clinicopathologic profiling and oncologic outcomes of secretory carcinoma of the breast
}

\author{
Piguo Gong ${ }^{1,5}$, Chen $\mathrm{Xia}^{2,5}$, Yifeng Yang ${ }^{1}$, Wang Lei ${ }^{3}$, Weiping Yang ${ }^{1}$, Junhua Yu ${ }^{1}$, Yishun $\mathrm{Ji}^{1}$, \\ Lijun $\operatorname{Ren}^{1}$ \& Fugui Ye ${ }^{4 \bowtie}$
}

Secretory carcinoma of the breast (SCB) is a rather rare entity of invasive breast cancer, the clinicopathologic characteristics and survival outcomes remain to be elaborated. A retrospective review was conducted in SEER database. A total of 190 SCB patients identified in SEER were eligible for inclusion in the analysis. Median age at diagnosis was 56 years (range 2-96 years). Both sexes and bilateral breast could be affected. Intriguingly, the incidence of SCB tended towards to decreasing in recent decades. Small tumor burden was observed with a mean tumor size of $2.13 \mathrm{~cm}$. In a subgroup with sufficient details, positive staining of estrogen receptor (ER) and progesterone receptor (PR) was $58 \%$ and $40 \%$, respectively. The vast majority of patients were of well to moderate differentiation (86.86\%) and negative regional lymph nodes involvement $(70.71 \%)$. Nearly half of the patients took radiotherapy and chemotherapy. Seniors were inclined to have an inferior breast cancer specific survival (BCSS) than their younger counterparts $(P=0.018)$. Patients underwent breast conserving surgery $(B C S)$ and radiotherapy had much better $B C S S$ than its mastectomy counterparts $(P=0.014)$. Collectively, SCB is a clinical indolent invasive breast cancer with excellent prognosis. BCS in conjunction with radiotherapy would be a rational alternative for this distinct entity.

Literature reported that rare cancer constitutes more than $20 \%$ of all cancer diagnoses annually ${ }^{1}$. To date, limited studies have been conducted on rare cancers, therefore many aspects are blind to clinicians, causing a worse prognosis for patients with rare cancers in comparison with common cancers. Another dilemma is that little is known regarding approaches to prevent and accurate diagnosis of many rare cancers ${ }^{2}$. Recent decades, tremendous advances in cancer genomics and cancer biology have resulted in great progress in cancer treatment profiles, leading to entrance the era of individualized precision medicine ${ }^{3}$. Consequently, focusing on the extremely rare variants of cancer would be of remarkable significance to improve the recognition and survival outcomes of the entire cancer community.

Secretory carcinoma of the breast (SCB) is a scarce but distinct subtype of breast malignancy, initially known as juvenile breast carcinoma by McDivitt and Stewart in $1966^{4}$, accounting for less than $0.15 \%$ of all breast cancers ${ }^{5}$. Usually, SCB has been reported as triple negative immunophenotype and positive staining for cytokeratins and EGFR, parallel to basal-like breast cancer, while the indolent clinical course as well as prolonged survival seem opposite to common triple negative breast cancer $(\mathrm{TNBC})^{6-8}$. Of note, few studies demonstrated that SCB could present with positivity for hormone receptors ${ }^{9-11}$. Cytogenetically, ETV6-NTRK3 gene fusion is deemed as the most characteristic feature of genomic alteration of SBC ${ }^{12,13}$. The ETV6-NTRK3 protein with transforming activity, could leads to constitutive activation of Ras-MAP kinase and phosphatidyl inositol-3-kinase-AKT pathways, participating in SCB carcinogenesis ${ }^{12}$. Thus, targeting ETV6-NTRK3 has been the priority of biomedical investigation. Given the exceeding rarity of SCB, there is not consensus regarding the optimal diagnostic criterium, treatment strategies and to what extent the excellent prognosis could be ascribed to clinicopathologic parameters.

\footnotetext{
${ }^{1}$ Department of Thyroid Breast Surgery, Qingdao Chengyang People's Hospital, Qingdao 266109, Shandong, China. ${ }^{2}$ Department of Medical Oncology, Hunan Cancer Hospital, The Affiliated Cancer Hospital of Xiangya School of Medicine, Central South University, Changsha 410013, China. ${ }^{3}$ Department of Breast Surgery, Affiliated Hospital of Jining Medical University, Jining 27200, Shandong, China. ${ }^{4}$ Key Laboratory of Breast Cancer in Shanghai, Department of Breast Surgery, Fudan University Shanghai Cancer Center, Shanghai 200032, China. ${ }^{5}$ These authors contributed equally: Piguo Gong and Chen Xia. ${ }^{\bowtie}$ email: yefugui87@163.com
} 

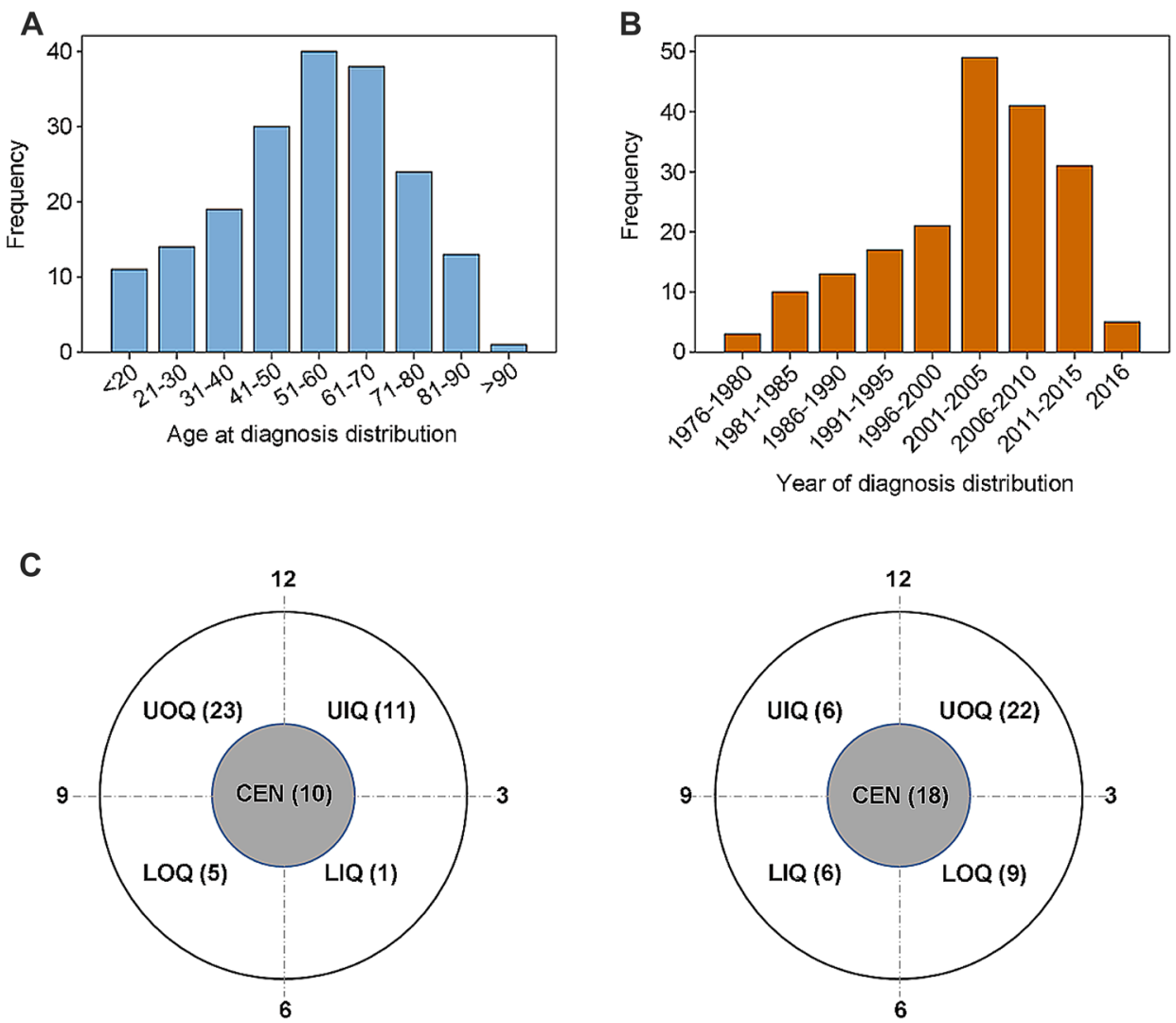

RIGHT BREAST

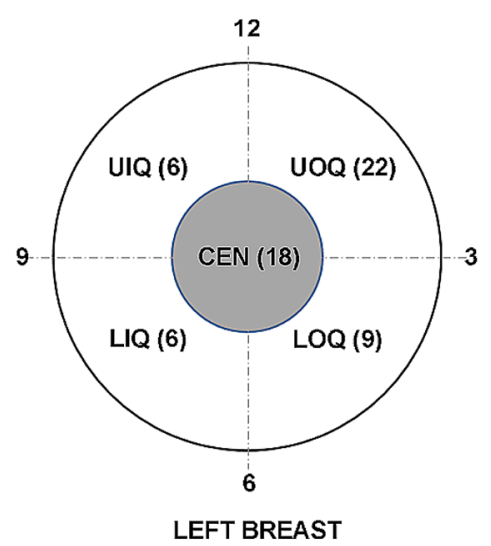

Figure 1. Clinicopathologic characteristics of study cohort. (A) Distribution of age at diagnosis. (B) Distribution of year of diagnosis. (C) Distribution of tumor location in the breasts. UIQ upper inner quadrant, $L I Q$ lower inner quadrant, $L O Q$ lower outer quadrant, $U O Q$ upper outer quadrant, CEN nipple-areola complex.

The large data volume in Survival, Epidemiology and End Results (SEER) program provides a well-established platform to investigate rare tumors. In this study, to help address the existing discrepancies and deep understand the nature of SCB, we examine the clinical and pathologic characteristics and long-term survival outcomes of SCB in a large number of cases. The findings probably shed light on our better understanding and management of SCB.

\section{Results}

Patient demographics. The demographic characteristics of the 190 SCB patients was summarized in Fig. 1. Median age at diagnosis was 56 years (range 2-96 years) (data not shown). Both sexes could be affected by SCB, with an approximate male to female ratio of 1:30 (data not shown). The proportion of patients diagnosed under the age of 30 years was $13.16 \%$ (Fig. 1A). Intriguingly, the number of patients was stable increasing as time went on, with a peak occurring at the beginning of twenty-first century, while declined sharply thereafter (Fig. 1B). Laterality was found to be comparable between the left and right breast, with 103 patients in left, 85 in right and 2 bilateral, respectively (data not shown). Primary tumor site was highest likely located in the upperouter quadrant (UOQ) irrespective of laterality (Fig. 1C).

Disease and treatment characteristics. In order to better understand the clinicopathologic features of SCB, a subgroup of 99 patients with sufficient and explicit information were derived and listed in Table 1 . Median age at diagnosis was 57 years (range: 8-89 years). Most patients were female white ethnicity and had tumors no more than $2 \mathrm{~cm}(\mathrm{~cm})$, with an average tumor size of $2.13 \mathrm{~cm}$. The vast majority of patients were of negative regional lymph nodes involvement (70.71\%) and well to moderate differentiation (86.86\%). Positive staining of estrogen receptor (ER) and progesterone receptor (PR) was $58 \%$ and $40 \%$, respectively. BCS was performed in 53 patients. The uptake of radiotherapy and chemotherapy was $45.45 \%$ and $41.41 \%$, respectively. Hormone therapy was unavailable in SEER database.

Oncologic outcomes. For all patients, the median follow-up time was 97 months (range 0-436 months). 5-year BCSS was 95.79\%, 10-year and 20-year BCSS both were 93.16\% (Fig. 2A). 5-year OS was 89.47\%, 10-year OS was $81.58 \%$ and 20 -year OS was $76.84 \%$ (Fig. 2B). Age-specific survivals were presented in (Fig. 2C,D). 


\begin{tabular}{|c|c|c|}
\hline \multirow[b]{2}{*}{ Characteristics } & \multicolumn{2}{|c|}{$\begin{array}{l}\text { All patients } \\
(\mathbf{n}=99)\end{array}$} \\
\hline & No. & $\%$ \\
\hline \multicolumn{3}{|c|}{ Age at diagnosis (years) } \\
\hline Median & \multicolumn{2}{|l|}{57} \\
\hline Range (IQR) & \multicolumn{2}{|c|}{$45-66$} \\
\hline \multicolumn{3}{|l|}{ Gender } \\
\hline Female & 95 & 95.96 \\
\hline Male & 4 & 4.04 \\
\hline \multicolumn{3}{|l|}{ Laterality } \\
\hline Left & 55 & 55.56 \\
\hline Right & 44 & 44.44 \\
\hline \multicolumn{3}{|l|}{ Ethnicity } \\
\hline White & 82 & 82.83 \\
\hline Black & 11 & 11.11 \\
\hline Other & 6 & 6.06 \\
\hline \multicolumn{3}{|c|}{ Pathologic tumor size } \\
\hline pT1 & 64 & 64.65 \\
\hline pT2 & 30 & 30.30 \\
\hline pT3 & 5 & 5.05 \\
\hline \multicolumn{3}{|l|}{ Nodal status } \\
\hline pNo & 70 & 70.71 \\
\hline $\mathrm{pN1}$ & 25 & 25.25 \\
\hline $\mathrm{pN} 2$ & 4 & 4.04 \\
\hline \multicolumn{3}{|l|}{ Nuclear grade } \\
\hline I & 41 & 41.41 \\
\hline II & 45 & 45.45 \\
\hline III & 13 & 13.13 \\
\hline \multicolumn{3}{|l|}{ ER status } \\
\hline Positive & 58 & 58.59 \\
\hline Negative & 41 & 41.41 \\
\hline \multicolumn{3}{|l|}{ PR status } \\
\hline Positive & 40 & 40.40 \\
\hline Negative & 59 & 59.60 \\
\hline \multicolumn{3}{|c|}{ Surgery modality } \\
\hline BCS & 53 & 53.54 \\
\hline Mastectomy & 46 & 46.46 \\
\hline \multicolumn{3}{|c|}{ Radiation therapy } \\
\hline Yes & 45 & 45.45 \\
\hline No & 54 & 54.55 \\
\hline \multicolumn{3}{|l|}{ Chemotherapy } \\
\hline Yes & 41 & 41.41 \\
\hline No/unknown & 58 & 58.59 \\
\hline
\end{tabular}

Table 1. Clinicopathologic characteristics and type of treatment of all patients included. $p T 1$ pathological tumor size $\leq 2 \mathrm{~cm}, p T 22 \mathrm{~cm}<$ pathological tumor size $\leq 5 \mathrm{~cm}, p T 3$ pathological tumor size $>5 \mathrm{~cm}, p N 0$ negative regional lymph node, $p N 1,1$ to 3 regional lymph node metastasis, $p N 24$ to 9 regional lymph node metastasis, Nuclear grade I well differentiation, Nuclear grade II moderate differentiation, Nuclear grade III poor differentiation, $E R$ estrogen receptor, $P R$ progesterone receptor, $B C S$ breast conserving surgery.

There was statistical significance in age-specific BCSS $(\mathrm{P}=0.018)$ and $\mathrm{OS}(\mathrm{P}<0.001)$, and worse prognosis was indicated with growing age.

For the subgroup cohort, the median follow-up time was 82 months (range 0-263 months). No statistical significance was observed with regard to BCSS $(\mathrm{P}=0.365)$ and $\mathrm{OS}(\mathrm{P}=0.603)$ categorized by hormone receptor status (Fig. 3A,B). Similar baseline characteristics of patients underwent BCS and radiotherapy and mastectomy were shown in Table 2. Although OS $(\mathrm{P}=0.185)$ was comparable between the two subsets, patients who underwent BCS and radiotherapy had much better BCSS than its mastectomy counterparts $(\mathrm{P}=0.014)$ (Fig. 3C,D). 

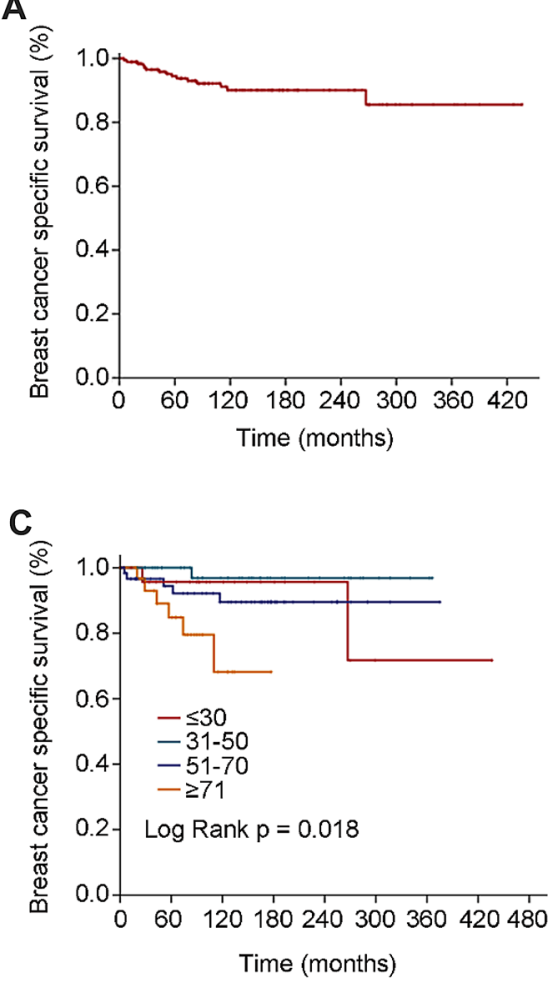

B
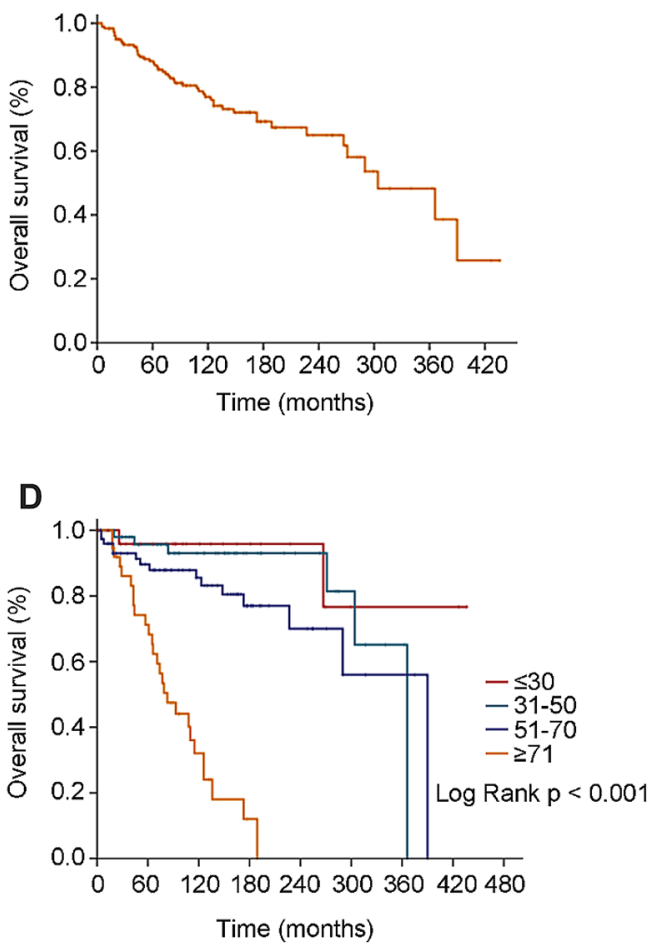

Figure 2. OS (overall survival) and BCSS (breast cancer specific survival) of study cohort. (A) Kaplan and Meier estimates BCSS. (B) Kaplan and Meier estimates of OS. (C) Kaplan and Meier estimates of age-related BCSS. (D) Kaplan and Meier estimates of age-related OS.

\section{Discussion}

SCB is a rare subtype of invasive breast malignancy, previous studies have been largely limited by case reports and small case series ${ }^{5}$. In the present study, we examined the demographics, disease characteristics, patterns of treatment and survival outcomes for patients diagnosed with SCB in a large-sized cohort derived from SEER database.

Despite initially discovered in juveniles, it was documented in a wide range of age groups and more frequently occur in adults later ${ }^{14}$. Till now, the two largest retrospective studies conducted by Horowitz et al. with 83 SCB patients published in $2012^{15}$. and Jacob et al. with 246 SCB patients published in $2016^{5}$ had illustrated a median age at diagnosis of 53 years in SEER database and 56 years in the National Cancer Data Base. Consistent with prior studies, median age at diagnosis was 56 years in this study. Additionally, Jacob et al. ${ }^{5}$ demonstrated a male to female ratio of 1:31, which was in agreement with that of 1:30 in our study. Nevertheless, a published experience of male to female ratio was approximately $1: 6^{16}$, which might be a result of small simple size. Confirming the previous report, the most common location of SCB was the UOQ of the breast, resembling that of invasive ductal carcinoma ${ }^{17}$.

Of note, great advances have been established in our understanding of biological nature of breast cancer, while the incidence of SCB was prone to continuously declining in recent decades. This was a new finding in the present study and some possible reasons would be rational to explain this phenomenon. Typically, SCB was presented with a slow-growing, painless, well-circumscribed, mobile mass, similar to that of benign epithelial proliferating lesions ${ }^{8,18,19}$, which may increase the possibility of misdiagnosis. Furthermore, non-specific and sparse ultrasound and mammographic findings associated with limited diagnostic value of ETV6-NTRK3 resulted in difficulty to differentiate diagnosis of $\mathrm{SCB}^{17,20-22}$.

There was no consistence on status of ER and PR receptor. The overwhelming majority of literature showed that SCB was negative for ER, PR, human epidermal growth factor (HER2), and positive for basal-cell markers, therefore could be classified as a peculiar subtype of TNBC ${ }^{6,23,24}$. Recent studies with large sample size concluded that SCB mimicked immunoprofile of hormone receptor positive cancer other than that of TNBC ${ }^{5,11,25}$, which was in stark contrast to the earlier reports. Our results were in support of the recent studies. In addition, other disease parameters were comparable with most previous studies, demonstrating that SCB usually presented as low grade, less likely to positive regional lymph node ${ }^{26,27}$.

Although axillary lymph node metastasis of SCB was described as high as approximately $15 \%-30 \%$ and patients with more than four lymph nodes were exclusively rare, the prognosis of SCB was excellent in published reports ${ }^{8,28}$. Horowitz et al. ${ }^{15}$ depicted that 5 -year BCSS was $94.4 \%$ and 10 -year BCSS was $91.4 \%$ in a study cohort of 83 patients. Recently, Li et al. ${ }^{11}$ uncovered that 5 -year OS was $93.2 \%$, and 10 -year OS was $88.6 \%$ in a study cohort of 44 patients. Our results were in favor of prior studies, with a 5-year BCSS was 95.79\%, 10-year 
A

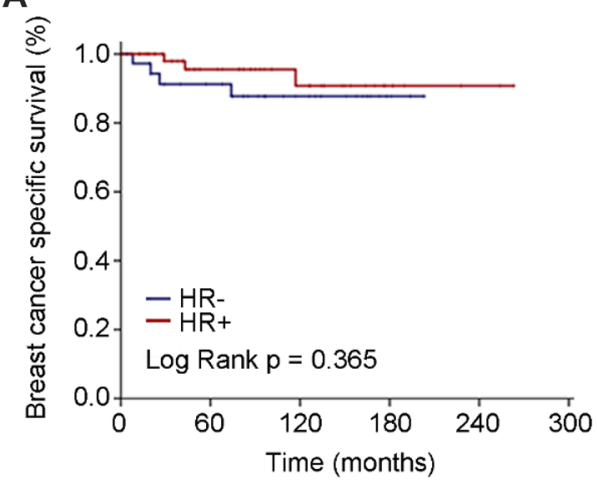

\section{C}

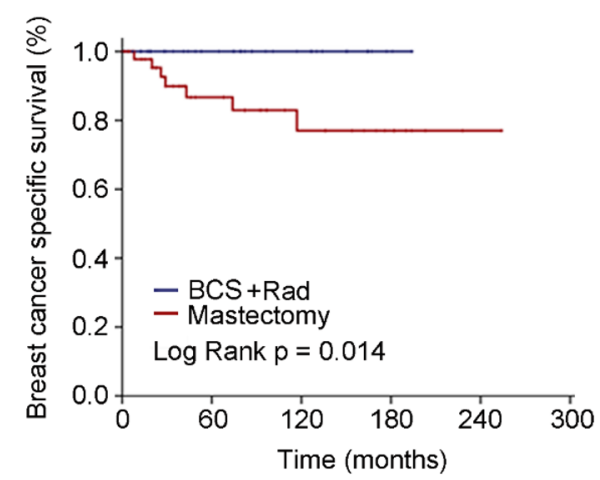

B

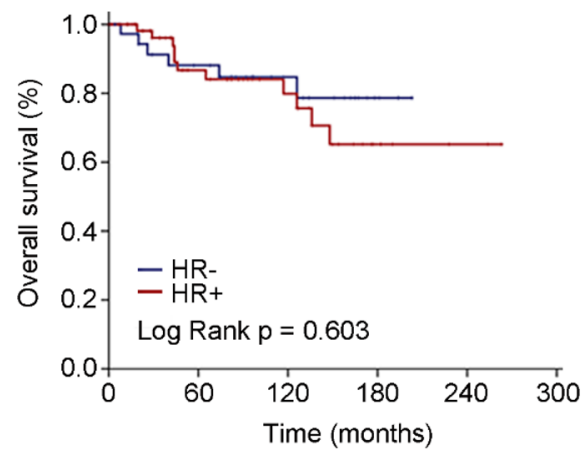

D

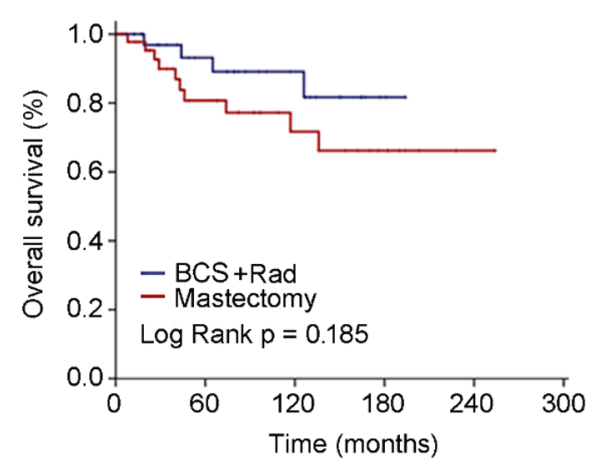

Figure 3. Survival outcomes categorized by hormone receptor and surgery modalities. (A) Kaplan and Meier estimates of BCSS (breast cancer specific survival) grouped by HR (hormone receptor) status. (B) Kaplan and Meier estimates of OS (overall survival) grouped by HR status. (C) Kaplan and Meier estimates of BCSS grouped by BCS (breast conserving surgery) and radiotherapy and mastectomy. (D) Kaplan and Meier estimates of OS grouped by BCS and radiotherapy and mastectomy.

and 20 -year BCSS both were $93.16 \%$ as well as 5 -year OS was $89.47 \%, 10$-year OS was $81.58 \%$ and 20 -year OS was $76.84 \%$. To a great extent, the excellent prognosis might be contributed to the early stage and mild clinicopathologic characteristics, aside from adjuvant systemic therapy. One should be cautious that distant metastasis could occur as late as 16 years after definitive local surgery ${ }^{29}$. Currently, the primary management of SCB was surgery, because of the indolent clinical course and fairly good prognosis. The impact beyond surgery on survival outcomes was largely unknown. We demonstrated that BCS and radiotherapy was superior to mastectomy with comparable OS but better BCSS. Genomic study of SCB indicated that mutational burden and copy number variant of secretory carcinoma of the breast is very $\operatorname{low}^{30}$, which supported the BCS and radiotherapy might be sufficient to the treatment of this rare subtype.

The current study has some limitations. One limitation of this study was that details of systematic therapy, such as hormonal therapy and targeted therapy, were unavailable. Thus, the contribution of systematic therapy on the outcomes needed to be further clarified in future study. Another limitation of this study was the subtype of patients based on immunohistochemistry was not fully available, namely the frequency of receptor status as well as distribution of subtype merited deeper investigation. Although limitations existed, our study indeed improved our understanding this rare entity of SCB patients, especially based on the well-balanced characteristics of compared subgroups.

In conclusion, our retrospective study with medium-sized sample size reinforced the indolent course and unexceptionable outcomes of SCB. Moreover, BCS and radiotherapy could be a reasonable treatment of SCB followed by adequate systematic therapy. More intensive supervision and follow-up should be emphasized to the senior SCB patients. Long-term surveillance should be emphasized and cooperative work are stringently needed to derived a precise conclusion. Future studies with large size and comprehensive details are urged to validate our results.

\section{Methods}

Study cohort. This study adopted data from the National Cancer Institute's Surveillance, Epidemiology, and End Results (SEER) 18 tumor registry database that was updated in November 2016. The SEER ${ }^{\star}$ Stat version 8.3.5 was used to case extraction. With site recode limited to breast, eligible patients were extracted based on the following inclusion criteria: pathologic diagnosis according to the International Classification of Disease for 


\begin{tabular}{|c|c|c|c|c|c|}
\hline \multirow[b]{2}{*}{ Characteristics } & \multicolumn{2}{|c|}{$\begin{array}{l}\text { BCS + Rad } \\
(\mathbf{n}=37)\end{array}$} & \multicolumn{2}{|c|}{$\begin{array}{l}\text { Mastectomy } \\
(\mathrm{n}=46)\end{array}$} & \multirow[b]{2}{*}{$p$-value } \\
\hline & No & $\%$ & No & $\%$ & \\
\hline \multicolumn{6}{|c|}{ Age at diagnosis (years) } \\
\hline Median & \multicolumn{2}{|l|}{58} & \multicolumn{2}{|l|}{57} & \\
\hline Range (IQR) & \multicolumn{2}{|c|}{$48-66$} & \multicolumn{2}{|c|}{$41-67$} & \\
\hline \multicolumn{5}{|l|}{ Gender } & 0.125 \\
\hline Female & 37 & 100.00 & 42 & 91.30 & \\
\hline Male & 0 & 0.00 & 4 & 8.70 & \\
\hline \multicolumn{5}{|l|}{ Laterality } & 0.840 \\
\hline Left & 20 & 54.05 & 27 & 58.70 & \\
\hline Right & 17 & 45.95 & 19 & 41.30 & \\
\hline \multicolumn{5}{|l|}{ Ethnicity } & 0.330 \\
\hline White & 32 & 86.49 & 37 & 80.43 & \\
\hline Black & 2 & 5.41 & 7 & 15.22 & \\
\hline Other & 3 & 8.11 & 2 & 4.35 & \\
\hline \multicolumn{5}{|c|}{ Pathologic tumor size } & 0.052 \\
\hline pT1 & 29 & 78.38 & 25 & 54.35 & \\
\hline pT2 & 6 & 16.22 & 18 & 39.13 & \\
\hline pT3 & 2 & 5.41 & 3 & 6.52 & \\
\hline \multicolumn{5}{|l|}{ Nodal status } & 0.722 \\
\hline $\mathrm{pN} 0$ & 28 & 75.68 & 29 & 63.04 & \\
\hline $\mathrm{pN} 1$ & 7 & 18.92 & 15 & 32.61 & \\
\hline $\mathrm{pN} 2$ & 2 & 5.41 & 2 & 4.35 & \\
\hline \multicolumn{5}{|l|}{ Nuclear grade } & 0.527 \\
\hline I & 17 & 45.95 & 17 & 36.96 & \\
\hline II & 17 & 45.95 & 22 & 47.83 & \\
\hline III & 3 & 8.11 & 7 & 15.22 & \\
\hline \multicolumn{5}{|l|}{ ER status } & 0.729 \\
\hline Positive & 24 & 64.86 & 27 & 58.70 & \\
\hline Negative & 13 & 35.14 & 19 & 41.30 & \\
\hline \multicolumn{5}{|l|}{ PR status } & 0.420 \\
\hline Positive & 17 & 45.95 & 16 & 34.78 & \\
\hline Negative & 20 & 54.05 & 30 & 65.22 & \\
\hline \multicolumn{5}{|l|}{ Chemotherapy } & 0.998 \\
\hline Yes & 17 & 45.95 & 20 & 43.48 & \\
\hline No/unknown & 20 & 54.05 & 26 & 56.52 & \\
\hline
\end{tabular}

Table 2. Clinicopathologic characteristics and chemotherapy of subgroups divided by type of surgery. $p T 1$ pathological tumor size $\leq 2 \mathrm{~cm}, p T 22 \mathrm{~cm}<$ pathological tumor size $\leq 5 \mathrm{~cm}, p T 3$ pathological tumor size $>5 \mathrm{~cm}$, $p N 0$ negative regional lymph node, $p N 1,1$ to 3 regional lymph node metastasis, $p N 24$ to 9 regional lymph node metastasis, Nuclear grade I well differentiation, Nuclear grade II moderate differentiation, Nuclear grade $I I I$ poor differentiation, $E R$ estrogen receptor, $P R$ progesterone receptor, $B C S$ breast conserving surgery.

Oncology, Third Edition (ICD-O-3), one primary site only and known age at diagnosis. Consequently, a total of 190 patients with pathologically confirmed invasive secretory carcinoma of the breast (ICD-O-3 8502/3) were identified and subjected to clinicopathologic characteristics and overall prognostic analyses. Patients with unknown information of variables of laterality, pathologic tumor size, nodal status, nuclear grade, ER status, PR status and type of surgery were excluded, thereafter 99 patients left to comprise a subgroup. The influence of clinicopathologic parameters and treatment interventions on survival outcomes were performed at the subgroup setting.

Definition of outcomes. Overall survival (OS), defined as the time from diagnosis to death from any cause. Cancer-specific survival (CSS), defined as the interval from initial diagnosis to death resulting from breast cancer.

Statistical analysis. Survival curves were established by using the method of Kaplan and Meier. The log-rank test was adopted to compare survival outcomes between different subgroups of patients. Categorical variables were compared using the Pearson's chi-square test or Fisher's exact test, as appropriate. All tests were 
two-sided, and $\mathrm{P}<0.05$ was considered statistically significant. Statistical analysis was performed by SPSS for windows (version 23.0, SPSS Inc., Chicago, IL, USA).

Ethics statement. This study was approved by the Ethical Committee of the Shanghai Cancer Center of Fudan University. We have submitted a request for the SEER data and complied with the sample data use agreement. The data released by the SEER database are publicly available and do not require informed patient consent. All methods were performed in accordance with relevant guidelines and regulations.

Received: 4 August 2020; Accepted: 8 July 2021

Published online: 19 July 2021

\section{References}

1. Keat, N. et al. International rare cancers initiative. Lancet Oncol. 14, 109-110 (2013).

2. Abbas-Aghababazadeh, F., Mo, Q. \& Fridley, B. L. Statistical genomics in rare cancer. Semin. Cancer Biol. 61, 1-10 (2020).

3. Greenwalt, I., Zaza, N., Das, S. \& Benjamin, D. L. Precision medicine and targeted therapies in breast cancer. Surg. Oncol. Clin. N. Am. 29, 51-62 (2020).

4. McDivitt, R. W. Breast carcinoma in children. JAMA 195, 388-390 (1966).

5. Jacob, J. D. et al. Rare breast cancer: 246 invasive secretory carcinomas from the National Cancer Data Base. J. Surg. Oncol. 113, 721-725 (2016).

6. Laé, M. et al. Secretory breast carcinomas with ETV6-NTRK3 fusion gene belong to the basal-like carcinoma spectrum. Mod. Pathol. 22, 291-298 (2009).

7. Jena, M. \& Shariff, S. Cytodiagnosis of secretory carcinoma of the breast: A report on two cases. Diagn. Cytopathol. 38, 921-924 (2010).

8. Li, D. et al. Secretory breast carcinoma: A clinicopathological and immunophenotypic study of 15 cases with a review of the literature. Mod. Pathol. 25, 567-575 (2012).

9. Yorozuya, K. et al. A case of estrogen receptor positive secretory carcinoma in a 9-year-old girl with ETV6-NTRK3 fusion gene. Jpn. J. Clin. Oncol. 42, 208-211 (2012).

10. Cabello, C. et al. Case report and review of the literature: Secretory breast cancer in a 13-year-old boy-10 years of follow up. Breast Cancer Res. Treat. 133, 813-820 (2012).

11. Li Lijuan, Wu., Nan, L. F., Lingmei, Li., Lijuan, W. \& Juntian, L. Clinicopathologic and molecular characteristics of 44 patients with pure secretory breast carcinoma. Cancer Biol. Med. 16, 139-146 (2019).

12. Tognon, C. et al. Expression of the ETV6-NTRK3 gene fusion as a primary event in human secretory breast carcinoma. Cancer Cell 2, 367-376 (2002).

13. Makretsov, N. et al. A fluorescence in situ hybridization study of ETV6-NTRK3 fusion gene in secretory breast carcinoma. Genes Chromosomes Cancer 40, 152-157 (2004).

14. Bond, S. J., Buchino, J. J., Nagaraj, H. S. \& McMasters, K. M. Sentinel lymph node biopsy in juvenile secretory carcinoma. J. Pediatr. Surg. 39, 120-121 (2004).

15. Horowitz, D. P., Sharma, C. S., Connolly, E., Gidea-Addeo, D. \& Deutsch, I. Secretory carcinoma of the breast: Results from the survival, epidemiology and end results database. Breast (Edinburgh, Scotland) 21, 350-353 (2012).

16. Serour, F., Gilad, A., Kopolovic, J. \& Krispin, M. Secretory breast cancer in childhood and adolescence: Report of a case and review of the literature. Med. Pediatr. Oncol. 20, 341-344 (1992).

17. Vasudev, P. \& Onuma, K. Secretory breast carcinoma: Unique, triple-negative carcinoma with a favorable prognosis and characteristic molecular expression. Arch. Pathol. Lab. Med. 135, 1606-1610 (2011).

18. Krausz, T., Jenkins, D., Grontoft, O., Pollock, D. J. \& Azzopardi, J. G. Secretory carcinoma of the breast in adults: Emphasis on late recurrence and metastasis. Histopathology 14, 25-36 (1989).

19. Amott, D. H., Masters, R. \& Moore, S. Secretory carcinoma of the breast. Breast J. 12, 183 (2006).

20. Paeng, M. H., Choi, H.-Y., Sung, S. H., Moon, B. I. \& Shim, S. S. Secretory carcinoma of the breast. J. Clin. Ultrasound 31, 425-429 (2003).

21. Hechtman, J. F. et al. Pan-Trk immunohistochemistry is an efficient and reliable screen for the detection of NTRK fusions. Am. J. Surg. Pathol. 41, 1547-1551 (2017).

22. Osako, T., Takeuchi, K., Horii, R., Iwase, T. \& Akiyama, F. Secretory carcinoma of the breast and its histopathological mimics: Value of markers for differential diagnosis. Histopathology 63, 509-519 (2013).

23. Lambros, M. B. K. et al. Genomic profile of a secretory breast cancer with an ETV6-NTRK3 duplication. J. Clin. Pathol. 62, 604-612 (2009).

24. Lange, A. \& Lo, H.-W. Inhibiting TRK proteins in clinical cancer therapy. Cancers 10, 105 (2018).

25. Jin, M.-S. et al. Integrated multi-omic analyses support distinguishing secretory carcinoma of the breast from basal-like triplenegative breast cancer. Proteomics Clin. Appl. 12, e1700125 (2018).

26. Diallo, R. et al. Secretory carcinoma of the breast: A distinct variant of invasive ductal carcinoma assessed by comparative genomic hybridization and immunohistochemistry. Hum. Pathol. 34, 1299-1305 (2003).

27. Toll, A., Joneja, U. \& Palazzo, J. Pathologic spectrum of secretory and mucinous breast lesions. Arch. Pathol. Lab. Med. 140, 644-650 (2016).

28. Vieni, S. et al. Secretory breast carcinoma with metastatic sentinel lymph node. World J. Surg. Oncol. 4, 88 (2006).

29. Tixier, H. et al. Long-term recurrence of secretory breast carcinoma with metastatic sentinel lymph nodes. Arch. of Gynecol. Obstetr. 283, 77-78 (2011).

30. Krings, G. et al. Genomic profiling of breast secretory carcinomas reveals distinct genetics from other breast cancers and similarity to mammary analog secretory carcinomas. Mod. Pathol. 30, 1086-1099 (2017).

\section{Author contributions}

Conception and design: P.G., C.X. and F.Y. Provision of study patients and methods: P.G., C.X. and F.Y. Collection of data: P.G. and C.X. Data analysis and interpretation: P.G., C.X. and F.Y. Manuscript writing: P.G., C.X. and F.Y. Final approval of manuscript: P.G., C.X., Y.Y., W.L., W.Y., J.Y., Y.J., L.R. and F.Y.

\section{Competing interests}

The authors declare no competing interests. 


\section{Additional information}

Correspondence and requests for materials should be addressed to F.Y.

Reprints and permissions information is available at www.nature.com/reprints.

Publisher's note Springer Nature remains neutral with regard to jurisdictional claims in published maps and institutional affiliations.

(c) (1) Open Access This article is licensed under a Creative Commons Attribution 4.0 International License, which permits use, sharing, adaptation, distribution and reproduction in any medium or format, as long as you give appropriate credit to the original author(s) and the source, provide a link to the Creative Commons licence, and indicate if changes were made. The images or other third party material in this article are included in the article's Creative Commons licence, unless indicated otherwise in a credit line to the material. If material is not included in the article's Creative Commons licence and your intended use is not permitted by statutory regulation or exceeds the permitted use, you will need to obtain permission directly from the copyright holder. To view a copy of this licence, visit http://creativecommons.org/licenses/by/4.0/.

(C) The Author(s) 2021 